\title{
An Adaptive Classification Framework for Data Streaming Anomaly Detection*
}

\author{
Menachem Domb, Guy Leshem \\ Ashkelon Academic College, Ashkelon, Israel
}

\begin{abstract}
Predicting the behavior of a system, we usually analyze its past data to discover common patterns and other classification artifacts. This process consumes considerable computational power and data storage. We propose an approach and a system, which requires much less resources without compromising prediction capabilities and accuracy. It employs three basic methods: common behavior graph, contour surrounding the graph, and entropy calculation methods. When the system is about to be implemented for a specific domain, the optimized combination of these three methods is considered, such that it fits the unique nature of the domain and its corresponding data type. In this work, we propose a framework and a process assisting system designers, finding the optimal methods for the case at hand. We demonstrate our approach with a case study of meteorological data collected over 15 years to classify and detect anomalies in new data.
\end{abstract}

Keywords: classification, anomaly detection, Internet of things (IoT)

\section{Introduction}

On Internet of things (IoT), sensor networks, and other applications, we often have sequential data from which we would like to extract useful knowledge. Given the nature of IoT applications, limited computational power and bandwidth resources prohibit us from large-scale data collection. Data may be large in its number of samples, its dimensionality, or both. In general, data aggregation can help to summarize data into a compressed set, where the compressed set preserves most of the critical information from the original data. Working on the compressed data can reduce the complexity of the sub-sequent knowledge discovery task to a traceable version, without compromising on performance at the task. One special case of data aggregation is sampling, which focuses on selecting only some of the samples from the original data uniformly or non-uniformly.

In this work, we attempt to find an optimal or close to optimal sampling approach to selecting data for knowledge discovery. We work on finding the best sampling strategy given sequential data that is generated from IoT applications.

\section{Dictionary Learning}

Dictionary learning (Mairal, Bach, Ponce, \& Sapiro, 2009) was known to be useful for extracting patterns hidden in data. We can apply dictionary learning to sequential data for tasks, such as natural language processing and video analysis, and as well as to non-sequential data. Given most IoT data that is collected in

\footnotetext{
${ }^{*}$ Acknowledgements: This research was funded by the Israeli Ministry of Science, Technology, and Space.

Menachem Domb, Dr., professor, Department of Computer Science, Ashkelon Academy College.

Guy Leshem, Dr., professor, Department of Computer Science, Ashkelon Academy College.
} 
sequential form, we can find a method that maintains a basis, where we have enough elements in the basis to describe most of the sequential patterns existent in the data. For instance, in an intelligent transportation system (ITS), we may use vehicle global position system (GPS) or other telematics traces to extract driving behavior from drivers. This can help us to collect a set of common sequential patterns from the sequential telematics data. In a smart home system, we may collect a set of most frequent activity trajectories for home members to be used for member authentication.

The problem we discuss here is finding an optimal sampling method given a set of time series, where we can have the same or similar information before and after the sampling reduction process, as far as the purpose the data serves in the context of the relevant application.

The input given is a set of time series: $D=\left\{d^{(1)}, d^{(2)}, \ldots, d^{(n)}\right\}$, with each time series of the form:

$$
d^{(i)}=\left\{d^{(i)}, d^{(i)}, \ldots, d^{(i)}, \ldots\right\}
$$

and each data point within a " $1,2, k$ " series is a pair (time stamp and numeric value): $d^{(i)}=\left(t^{(i)}, x^{(i)}\right)$ archive and query massive time series streams. They apply " $k, k, k$ ".

The required output is an optimal set $D_{w}=\left\{a_{1}, a_{2}, \ldots, a_{m}\right\}$, where $a i$ can be any sampling artifact, such as a minimal data set, trends, graphs, measurements, or rules, which strongly represents and supports the purpose of collecting the original data set $D$.

How to judge whether $D_{w}$ indeed represents and supports the purpose of collecting the original data set $D$, depends on the intended application. There are many known data reduction techniques, which mainly enable restoring the original data set from the reduced one. Among these are compression and compaction routines and dictionary methods. The purpose is not to be able to restore the original data, but rather to serve best the purpose for which the data was collected.

We focus on two possible purposes. Given the sequential data, one purpose could be classification, and the other could be time series prediction.

\section{Series Classification}

In this problem, we consider the set $D_{\mathcal{w}}$ and the full data set $D$ as containing the same information, if they produce the same classifier (Dietterich, 2002). That is, if $f(d)=f_{w}(d) \in\{-1,1\}$ for every new data series $d$, where $f$ is a classifier learned from $D$ and $f_{w}$ is a classifier based on $D_{w}$. For instance, we can judge whether a series of yearly temperatures represent an El Nino (EN) year or not, or whether a series of sensor data is characteristic of a suspected intrusion or not.

\section{Time Series Prediction}

Here, we consider two sets $D$ and $D_{w}$ as containing the same (or similar) information if both can predict the future pattern of an initial series $d$. That is, we can use either $D$ or $D w$ to predict a future item $d n$ with similar accuracy (Mairal et al, 2009).

In this work, we deal mainly with classification. The rest of this article is organized as follows: In Section II, we discuss the literature review; we detail the proposed method for creating $D w$ from the given $D$, namely, by creating what we call a contour around an averaged series in Section III; Section IV introduces the data we used and shows experimental results of the proposed method; and we conclude our work and outline future directions in Section V. 


\section{Literature Review}

Real-world data typically contains repeated and periodic patterns. This suggests that the data can be effectively represented and compressed using only a few co-efficients of an appropriate basis. However, distance estimation when the data is represented using different sets of co-efficients is still a largely unexplored area. Vlachos, Freris, and Kyrillidis (2015) formulated the problem of estimating lower/upper distance bounds as an optimization problem and establish the properties of optimal solutions to develop an algorithm which obtains an exact solution to the problem. It is applicable to any sequential or high-dimensional data and any orthogonal data transformation. Sakurada and Yairi (2014) used auto-encoders with non-linear dimensionality reduction for the anomaly detection task. Their work demonstrates the ability to detect subtle anomalies where linear principal component analysis (PCA) fails. Reeves, Liu, Nath, and Zhao (2009) presented framework to multi-scale analysis to decompose time series and to obtain sparse representations in various domains.

Chilimbi and Hirzel (2002) implemented a dynamic pre-fetching scheme that operates in several phases. Firstly, profiling which gathers a temporal data reference profile from a running program. Secondly, an algorithm for extracting hot data streams, which are data reference sequences that frequently repeat in the same order. Thirdly, dynamically injecting code at appropriate program points to detect and pre-fetch these hot data streams. Finally, the process enters the hibernation phase where the program continues to execute with the added pre-fetch instructions. At the end, the program is optimized to remove the inserted checks and pre-fetch instructions, and control returns to the profiling phase. Lane and Brodley (1999) presented an approach based on instance-based learning (IBL) techniques.

Classification boundaries are selected from a posteriori characterization of valid user behaviors, coupled with a domain heuristic. An empirical evaluation of the approach on user command data demonstrates that we can accurately differentiate the profiled user from alternative users when the available features encode sufficient information. Furthermore, they demonstrate that the system detects anomalous conditions quickly - an important quality for reducing potential damage by a malicious user. They present several techniques for reducing data storage requirements of the user profile, including instance-selection methods and clustering. An empirical evaluation shows that a new greedy clustering algorithm reduces the size of the user model by $70 \%$, with only a small loss in accuracy. Kasiviswanathan, Melville, Banerjee, and Sindhwani (2011) addressed the problem of identifying emerging topics using dictionary learning. They propose a two-stage approach based on detection and clustering of novel user-generated content to derive a scalable approach by using the alternating directions method to solve the resulting optimization problems. Mairal et al. (2009) focus on modeling data vectors as sparse linear combinations of basic elements generating a generic dictionary, and then adapt it to specific data. They propose an optimization algorithm for dictionary learning, based on stochastic approximations, which scale up gracefully to large data sets of training samples.

Aldroubi, Cabrelli, and Molter (2008) proved that given a set of data vectors in a Hilbert space, there existed an optimal collection of sub-spaces minimizing the sum of the square of the distances between each vector and its closest sub-space in the collection. This collection of sub-spaces gives the best sparse representation for the given data and provides an optimal model for sampling in union of sub-spaces. Rubinstein, Bruckstein, and Elad (2010) surveyed the various options up to the most recent contributions and structures. Cherian, Sra, and Papanikolopoulos (2011) proposed learning over-complete dictionary models where the signal can have both Gaussian and (sparse) Laplacian noise. Dictionary learning in this setting leads 
to a difficult non-convex optimization problem, which is further exacerbated by large input datasets. Duarte-Carvajalino and Sapiro (2008) introduced a framework for the joint design and optimization of the non-parametric dictionary and the sensing matrix. They demonstrate the use of random sensing matrices and those matrices that are optimized independently of the learning of the dictionary. The presentation of the framework and its efficient numerical optimization is complemented with classical image data sets.

\section{The Problem Statement}

The common underlying idea of the reviewed approaches is the definition of the problem they are aiming to solve. The problem attempted to be solved is optimizing the size of the collected sampling data, so that it keeps the proper balance between the quantity of sampling data and the information extracted from it. Hence, looking for ways to manipulate the sampling data and reduce it to a manageable size, and extract the desired information from it, as it is able to do using the original sampling data.

The problem statement focuses on extracting concepts, methods, rules, and measurements, so that at the end of the process, the original sampling data becomes redundant and need no longer be stored. However, to keep improving and adjusting the extracted artifacts to natural changes in the behavior of the sampled mechanism, we incorporate in the approach an ongoing learning process. In addition, in the study, we concentrate on time dependent streaming sampling data, divided by fixed periods, so that we can repeat the analysis process for each period/cycle. Thus, while there are many classification algorithms using time series sampling, the aim here is not to compare the performance of yet another classifier, rather the purpose is to present a flexible method for compactly representing the data with several parameters which can be chosen and adjusted. Lee, Pao, Shih, Lin, and Chen (2016) proposed a similar compressed representation for time series, referred to as an "envelope." However, while they used just the standard deviation $(S D)$ and the common mean methods as a fixed definer of the compressed representation, we suggest a method independent framework allowing a flexible adaptation of the contour to the nature of the given domain.

Indeed, some of the reviewed work, such as Reeves et al. (2009) could be revisited and be adjusted to the problem statement and so became a valid alternative to the approach we presented.

\section{Contour Based Approach}

As mentioned, the proposal assumes periodic data sampling and extraction of logical artifacts at period level. In a glance, we analyze sampling data collected over several periods. We divide the period to time-units. For example, for a year period, we divide it into daily time-units. For each time-unit, we extract one value representing it. This is done by averaging the samples collected during the time-unit. In the example, we may calculate the average value of all samples of that day. We may also decide to select one of the samples to represent the day, e.g., the first or last sample. We then calculate the average value for each time-unit out of the collected values for the same time-unit in all periods, resulting with an average value for a given time-unit. We repeat this process for all time-units in the period and get a graph representing the average values for an average and common period.

Assuming we have the average graph line for an average period, we now calculate the contour around this average. The generated contour represents the standard range of values, such that an unanalyzed period can be compared to this contour. If its graph value is completely within the contour, it means that this period is a standard period. If it is completely out of the contour then, it is purely an out of standard. In case, the sections 
of the graph are within the contour, while other sections are out of it, we use an entropy measure to calculate the overall "distance" of the given period from the standard contour. Assuming an existing entropy threshold, we can decide if the period in a standard period or not.

We apply the same concept at the unit level and decide if a specific time-unit in a period is within the standard or not. This specific check is relevant, for example, to anomaly detection of IoT behavior.

In summary, the entire process is based on three key elements: average graph per period, contour around the average graph, and an entropy value representing the overall distance of a period from the contour.

Each of these elements - average, contour, and entropy — can be one of several possibilities. For example, as an average measure, the straightforward arithmetic average could be used. But the geometric, harmonic, or any other, could just as well be evaluated and used. For the contour, a simplistic choice would be minimum and maximum (min-max) values. Alternatively, $S D$ or confidence interval (CI) could be employed. These three elements affect each other, and every choice of such a triplet—average, contour, and entropy—will produce a different behavior of the compressed classifier. The object is to find the best such triplet, to be able to disregard the original data after extracting the representing contour from it, without compromising on the ability to successfully analyze future series. In the work, we consistently use the arithmetic average and classical entropy, and focus on finding the best choice of contour.

\section{Finding the Optimal Contour}

We begin with a supervised learning approach, for classification, in which each time series is labeled as one of two classes. To demonstrate, using the data set from the experiments, the time series are year-long recordings of temperature samplings, labeled as positive, if the corresponding year was an EN year, or negative otherwise.

We now describe in detail the process of building the classifier, with emphasis on finding the optimal contour. Optimality here is in the sense of best predictive output that is, finding the contour parameters which result in the classifier with the best results:

1. Collecting several cycles of data from a given sensor. For example, daily temperature collected over a year is considered one cycle. Each record in the cycle contains a time stamp and a numeric value. These cycles are labeled positive or negative, e.g., "EN year" and "Non-El-Niño (NEN) year;"

2. Verifying that the collected data truly represents the sensor's normal and abnormal behavior;

3. Calculating the average cycle for each class, using a chosen average function, by averaging at every time stamp over all the cycles in the respective class;

4. Calculating the contour around the average, for each class, using different contour functions - min-max, $S D$, and CI - by taking at every time stamp, the calculated interval above and below the average.

The process of constructing the best contour is described in Figure 1. We begin with raw data collected during $N$ periods generating corresponding data cycles, where each record corresponds to a value related to a specific time-unit. For example, a cycle is a year and a time-unit is a day. These cycles have already been classified positive or negative according to some classification criteria. These classified cycles will later be used to determine the best contour among several proposed contours. This data is filtered and cleansed by removing defected records and merging records to get one record per time-unit. After this preliminary data preparation, we commence with the process. 


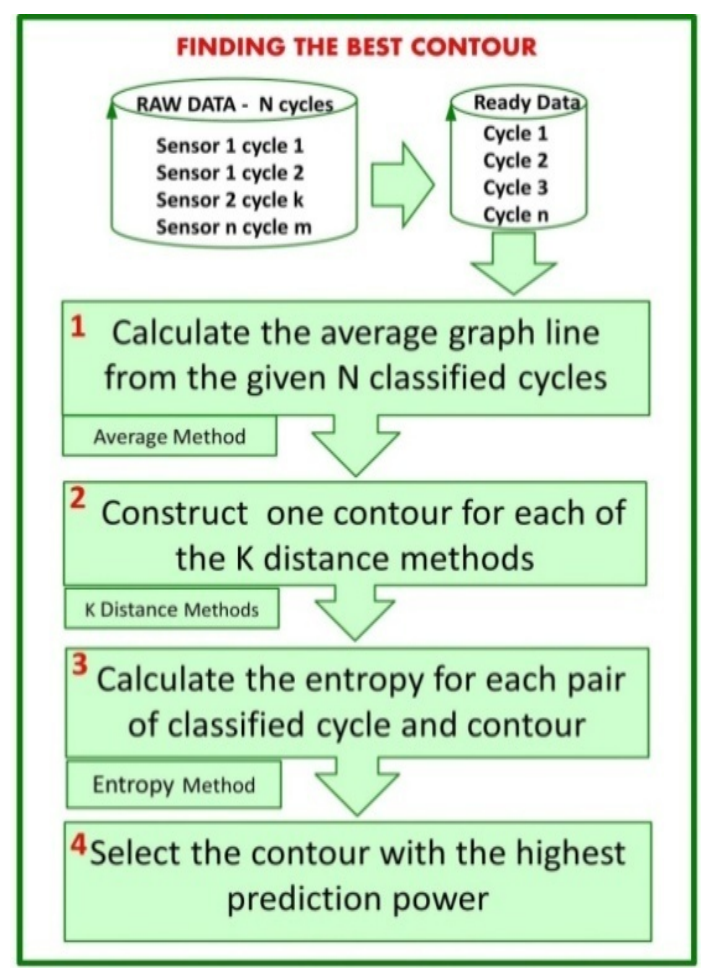

Figure 1. Process of finding the optimal contour.

The process is divided into four stages. In Stage One, we use a selected average method and calculate the average graph line representing the $N$ given cycles. This is done horizontally by calculating the average of the values related to the same time-unit across all $N$ cycles. For example, we calculate the average of the values for January 1 st across the various years. Doing so for all time-units will generate the average graph line. In Stage Two, we select several distance calculation methods and for each method we construct its associated contour. This is done by calculating the distance value for each distance method, e.g., min-max difference, $S D$, and CI. Taking the distance value, we add and subtract it from the average line to get the contour around the average. We repeat this process for all distance methods. At this stage, we have constructed several contours around the average line. The goal now is to select the contour which is most effective in classifying unclassified cycles. This is done in Stages Three and Four. In Stage Three, we calculate the prediction power for each contour and select the one with the highest prediction power. This is done by summing, for each contour, the number of cases its prediction was right, and calculating the average entropy of these correctly classified cycles. We do the same for wrong predictions. In Stage Four, we use one entropy method with an associated threshold value. An unclassified cycle with an entropy value lower than the threshold will be classified positive and negative otherwise. For each contour, we calculate the entropy of the given classified cycles. The result is a set of entropy values, where some are below the threshold while other are above it.

We repeat this for all classified cycles. We then sum up the number of correct predictions and their total entropies. We do the same for wrong predictions. We then subtract the total wrong numbers from the correct numbers. To get the final measure, we multiply these two numbers and get a measure representing the prediction power of the corresponding contour. We repeat this process for all the constructed contours and we will select the contour having the highest prediction power. 


\section{Calculating the Entropy}

The entropy of a period, given a contour, is calculated as follows:

1. Marking for every time stamp whether the cycle's value at that timestamp is below, within, or above the contour;

2. Calculating the frequency of each of these three possibilities — below $\left(p_{1}\right)$, within $\left(p_{2}\right)$, and above $\left(p_{3}\right)$;

3. Using these as a ternary probability distribution, its entropy is calculated according to the formula: $p_{1}$ $\log { }^{(p 1)}+p_{2} \log { }^{(p 2)}+p_{3} \log { }^{(p 3)}$.

The entropy measure is expected to return its minimum value, i.e., 0. At the two extreme cases: When the cycle graph is entirely contained within the contour, and when the cycle graph lies entirely outside of the contour. The first case fits in with the model, since a low entropy value would lead to the correct conclusion that the cycle is of the type which the contour represents. However, the second case would seemingly cause a false positive classification. We are not bothered by this, since we do not expect, such as harp exclusion from the contour. Rather, all cycles are expected to fall mostly within the contour, and those which diverge enough from the contour, will have a high entropy value which will lead to the right conclusion. Calculation of the entropy threshold is an outcome of a sensitive analysis we conduct on the entropy value.

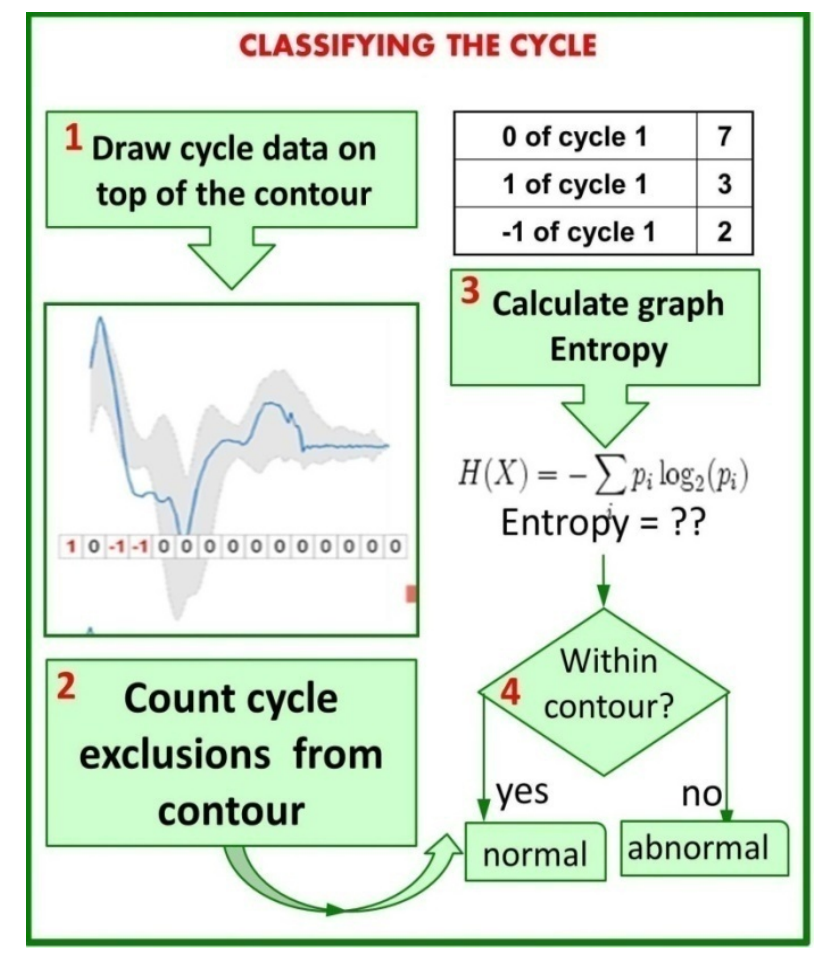

Figure 2. Classifying a cycle.

\section{Classifying a Cycle/Period}

Figure 2 describes the process of classifying new, unlabeled data cycles, as we now elaborate:

1. Applying the given data cycle to the contour, matching up according to time stamps;

2. Noting for each time stamp whether the data point is below the contour, within it, or above it;

3. Marking these cases respectively as $-1,0$, and +1 ;

4. Calculating the frequencies of each of the three values: $-1(p 1), 0(p 2)$, and $+1(p 3)$; 
5. Calculating the entropy of the distribution defined by $p 1, p 2$, and $p 3$;

6. Classifying as belonging to the contour, if the entropy is below the threshold determined in the learning phase.

\section{Advantages of Proposed Technique}

The proposed technique for defining a contour as a sparse representation of the data has several advantages over other existing methods.

Firstly, the technique is a family of sampling methods, where each member of this family is defined by the three parameters described above: Every choice of average calculation, contour definition, and entropy formula defines a method which can be applied to the data. It is reasonable to expect that different data sets will require different parameters for the best sampling. Different combinations can be tested and evaluated, before being applied to real-time data, to ensure optimal treatment of the data. It is also possible to dissect the data cycles into different time intervals, and use different parameters for each section, for data which requires such temporal differentiation. The technique we propose is therefore flexible and adjustable, made to fit for every given data set.

Secondly, this technique can be applied not only for classification but also for time series prediction: Data cycles can be diagnosed at real-time, before they are completed and classified, by identifying trends in the cycle graph, as compared to the class contours, and by calculating and updating the entropy as the data streams in.

Thirdly, the technique can be used to evaluate reliability of data online. In cases of high fluctuations or sharp changes in the cycle graph, which do not conform to either of the two class contours, it can be suspected that data reliability has been compromised. This can hint to the fact that the sensor is damaged or that there has been a security breach.

Fourthly, the approach allows self-learning and automatic adjustments in case the common behavior changes and a new standard have been established. As mentioned, trends in the cycle graph and contour can be identified within a cycle. However, such trends can be repeated and become the normal case. In such a case, the system can identify the situation based on a pre-defined criterion, and then an adjusted cycle graph may be proposed, such that the number of exceptions is reduced and is manageable.

Fifthly, occasionally, we may run a post mortem and check the system reaction to the actual behavior, and so try to adjust system parameters accordingly.

\section{Anomaly Detection for IoT Security}

IoT devices generate time related data, i.e., structured records containing a time stamp and one or more numeric values. In many cases, we can identify recurrent time frames where the system behavior has a repeating format. Hence, IoT data is of a structure to which the contour approach is highly applicable.

In IoT security, we are looking for common data patterns and quantitative measurements. Based on the identified patterns and measurements, we can extract logical rules that will be executed once an exception is discovered. An exception may be any violation of predefined patterns, measurements, and other parameters which represent normal, standard, and permitted behavior. In IoT, there is an abundance of possible patterns, starting with column level patterns up to a super internet controlling several IoT networks. The goal is to find the methods and tools to define standard patterns and how they can be identified. Once this is done, we can apply the contour method. In the work, we show a two-dimension contour. Using the same concept, we can 
expand it to be a multi dimension contour. This case is common where there is a dependency among several columns within one record and the same applies for the case where there are dependencies among networks of IoT systems.

\section{Case Study}

In the following case study, we do not present a detailed quantitative analysis. Rather, we show only the results which are relevant to this specific data set, since the aim is not to go into the details, but rather to show in a general way that this is a viable approach. A more rigorous study of the data will be performed in the future. We used meteorological data collected about EN years (positive class) and NEN years (negative class) from 1980 to 1998. For the positive contours, we took data from the EN years 1982, 1983, 1987, 1988, 1991, and 1992. All other years in the range were NEN years. Of all the data recorded, we regarded only the time stamp and the sea-level temperature. We tested three methods for generating contours: (a) max-min over all cycles; (b) average cycle $\pm S D$; and (c) $\mathrm{CI}$.

Figures 3 and 4 show the contours for NEN years. Figure 3 shows in black, the NEN contour according to the average $\pm S D$, and depicts how EN years diverge from this contour, as compared to the NEN year-1995. The 1992 and 1988 (EN years) show clear divergence from the contour while 1995 (a NEN) is more contained within the contour. This is nicely captured by the entropy values, which for 1992 was 0.4266 and for 1988 was 0.3857-above the threshold, leading to the conclusion that they are not NEN years-while for 1995 the entropy was 0.3631 - significantly lower than those of the EN years, leading to the correct conclusion that 1995 is indeed a NEN year.

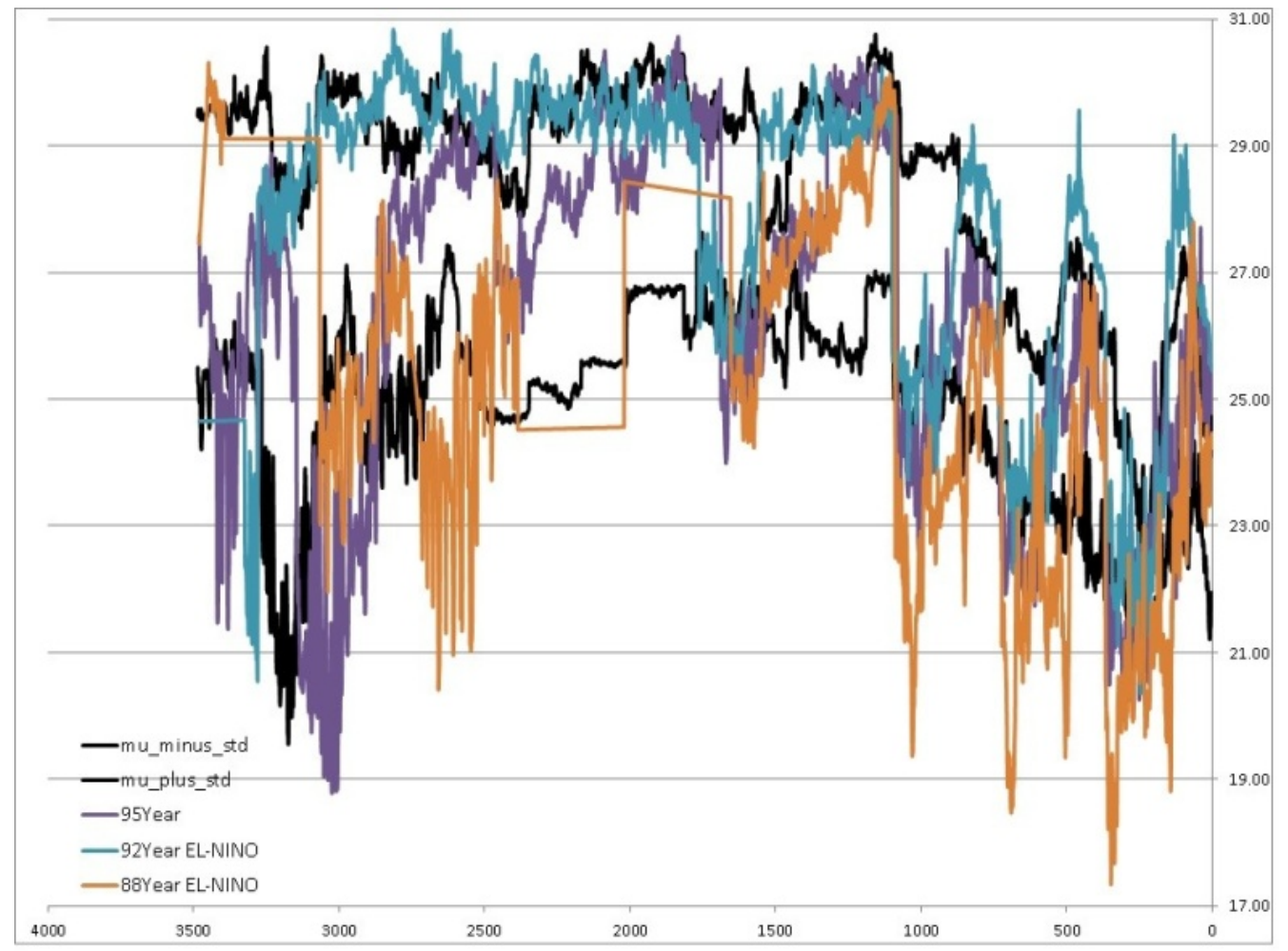

Figure 3. EN cycles on NEN average $\pm S D$ contour. 


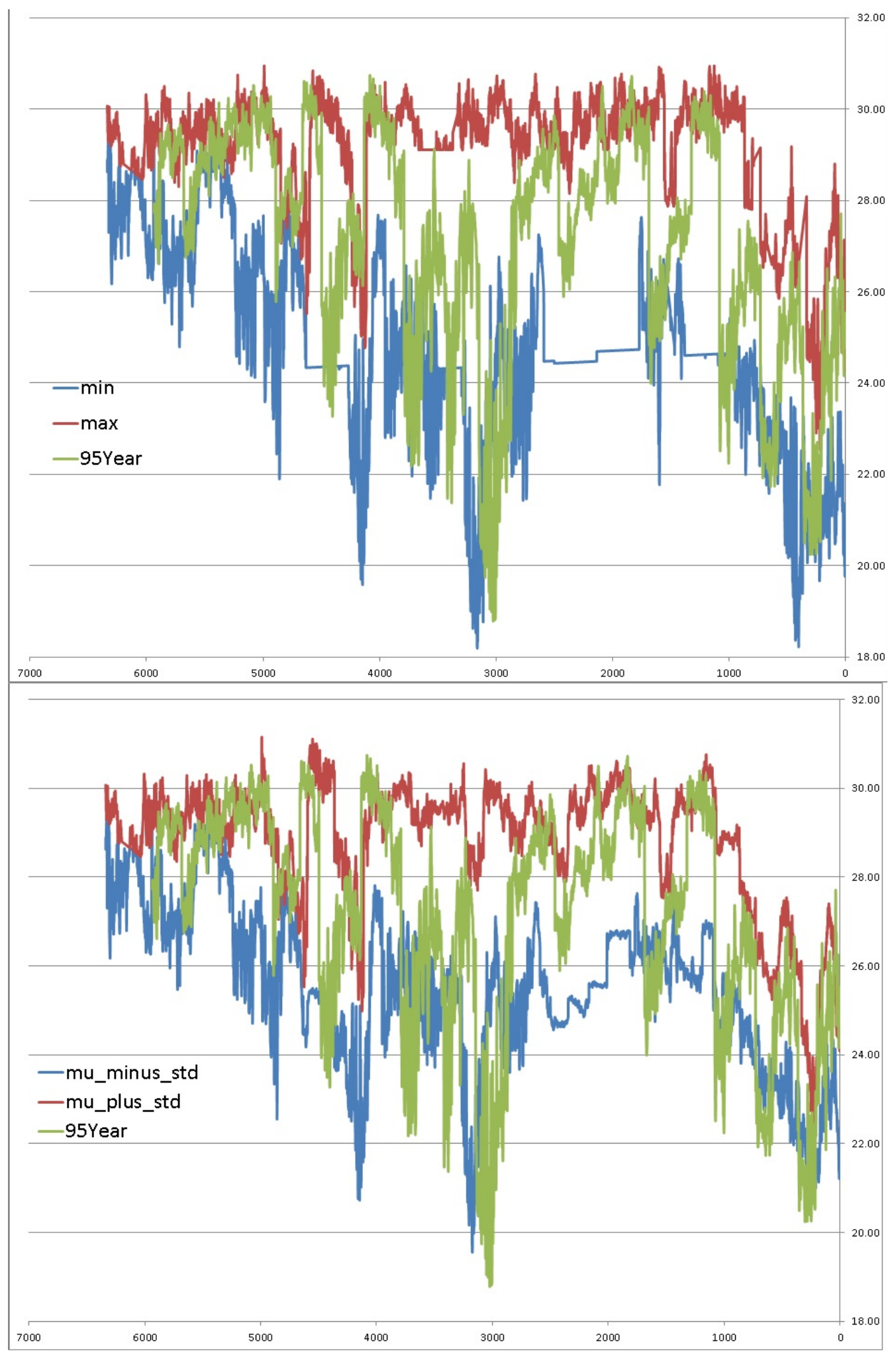

Figure 4. NEN contours - min-max and $S D$.

Figure 4 shows two contours: the min-max contour and the average $\pm S D$ contour. The $Y$-axis in these graphs is the temperature value, and the $X$-axis is the time. Within each contour, the year 1995 (a NEN year) is 
graphed. Its entropy is 0.3631 for the average $S D$ contour, and 0.2932 for the min-max contour. Both are the threshold, which leads to the correct conclusion that it should indeed be classified as NEN.

In the case study, we compared the contours constructed by using the average graph $\pm S D$ and the average graph \pm min-max. For the $S D$ contour, we got a significant entropy value difference between a classified EN case and a NEN case. In comparison, the min-max contour resulted in close values of entropy for the EN cycle and the NEN cycle. This means, that the ability to differentiate between two extreme situations, using entropy, depends on the parameter used to build the contour. Hence, in general, we must explore other parameters for defining the distance between the average lines to the contour borderlines. For every such calculation, we calculate the corresponding entropy for the two extreme values. Then, test it with already classified data and see if the calculated entropy allows the clear differentiation between positive and negative cases.

\section{Conclusions and Future Work}

In this study, we dealt with the classification problem of an unclassified cycle of streaming data, which is common in IoT. Classification has recently gained much attention due to rising IoT security issues and threats. We introduced the contour approach to draw the borders around the standard area representing a specific class. In case of an unclassified cycle, we measure its distance from the contour using an entropy formula. Then, we compare the result to a predefined threshold. If the entropy value is below the threshold, the cycle is of the same class.

We propose a process for constructing the best contour that most probably classifies to the correct underlying class. The process is based on three measurement methods: averae, distance, and entropy. For each method, there are several alternate formulas we may use. Each combination of these three methods may result in a different contour and hence, different entropy value, for the same unclassified cycle. To select the best contour, we use several already classified cycles and repeat the classification process using a different method triplet combination, each time. We select the combination with the maximum difference between positive and negative values. In the current study, we have dealt mainly with the problem of classification. However, as mentioned above, the contour method can be used for time series prediction as well. Even when the device records data which falls within the contour, trends can be discovered: We may note an inclination of values, such that an extrapolation shows the cycle leaving the contour in the future. This can be noticed by entropy values which are still low, but creeping upwards toward the threshold. In such a case, appropriate action can be taken ahead of time, to deal with the situation. For example, in the case of the EN data, temperature recordings along the year might be showing a consistent shift, enabling the prediction that the graph will leave the contour, and thus, the year will be declared as an EN year, before standard methods would be able to do this.

In addition to the initial construction of the class contours from the given data, we suggest ongoing improvements of the initial contours: With a periodic analysis of recent data, we may recalculate the class averages and their contours, so as to refine and revise the contours, for improved classification performance.

As mentioned above, this paper presents a general framework, without going into a detailed analysis. In future work, we intend to apply the method to other data sets within a more rigorous experimental setup. Doing so will enable us to improve the contour approach, in several aspects, such as determining the minimal number of classified cycles required to define the best contour, expanding the use of the contour to discover early trends or discover significant changes in behavior and adjusting the contour accordingly, exploring the possibility of 
dividing one cycle into several segments, and associating a different contour method to each segment. Classification is only one-way to cope with normally detection of data streaming. In future work, we will expand the approach to generate rules and measurements for discovering additional anomalies.

\section{References}

Aldroubi, A., Cabrelli, C., \& Molter, U. (2008). Optimal non-linear models for sparsity and sampling. Journal of Fourier Analysis and Applications, 14(5-6), 793-812.

Cherian, A., Sra, S., \& Papanikolopoulos, N. (2011). Denoising sparse noise via online dictionary learning. In 2011 IEEE International Conference on Acoustics, Speech and Signal Processing (ICASSP) (pp. 2060-2063). San Francisco, USA.

Chilimbi, T. M., \& Hirzel, M. (2002). Dynamic hot data stream prefetching for general-purpose programs. ACM SIGPLAN Notices, 37(5), 199-209.

Dietterich, T. G. (2002). Machine learning for sequential data: A review. In Joint IAPR International Workshops on Statistical Techniques in Pattern Recognition (SPR) and Structural and Syntactic Pattern Recognition (SSPR) (pp. 15-30). American: Springer.

Duarte-Carvajalino, J. M., \& Sapiro, G. (2008). Learning to sense sparse signals: Simultaneous sensing matrix and specifying dictionary optimization. IEEE Trans Image Process, 18(7), 1395-408. doi: 10.1109

Kasiviswanathan, S. P., Melville, P., Banerjee, A., \& Sindhwani, V. (2011). Emerging topic detection using dictionary learning. In Proceedings of the 20th ACM International Conference on Information and Knowledge Management (pp. 745-754). Athens, Greece.

Lane, T., \& Brodley, C. E. (1999). Temporal sequence learning and data reduction for anomaly detection. ACM Transactions on Information and System Security (TISSEC), 2(3), 295-331.

Lee, Y. J., Pao, H. K., Shih, S. H., Lin, J. Y., \& Chen, X. R. (2016). Compressed learning for time series classification. In 2016 IEEE Conference on Big Data. Washington, D.C.

Mairal, J., Bach, F., Ponce, J., \& Sapiro, G. (2009). Online dictionary learning for sparse coding. In Proceedings of The 26th Annual International Conference on Machine Learning (pp. 689-696). Nuremberg, Germany.

Reeves, G., Liu, J., Nath, S., \& Zhao, F. (2009). Managing massive time series streams with multi-scale compressed trickles. Proceedings of the VLDB Endowment, 2(1), 97-108.

Rubinstein, R., Bruckstein, A. M., \& Elad, M. (2010). Dictionaries for sparse representation modeling. Proceedings of the IEEE, 98(6), 1045-1057.

Sakurada, M., \& Yairi, T. (2014). Anomaly detection using auto encoders with nonlinear dimensionality reduction. In Proceedings of the MLSDA 2014 2nd Workshop on Machine Learning for Sensory Data Analysis (p. 4). Arizona, USA.

Vlachos, M., Freris N. M., \& A. Kyrillidis (2015). Compressive mining: fast and optimal data mining in the compressed domain. The VLDB Journal, 24(1), 1-24. 